\title{
Espacialização do grau de vulnerabilidade dos solos da Bacia do Rio Preto, Bahia, Brasil
}

\section{Nayara Caroline Moreira Leopoldo ${ }^{1}$, Bruna de Freitas Iwata $^{2}$, Tancio Gutier Ailan Costa ${ }^{3}$, Juliana Vogado Coelho $^{1}$, Gleide Ellen dos Santos Clementino ${ }^{1}$ e Cristian Epifânio de Toledo ${ }^{4}$}

\author{
${ }^{1}$ Instituto Federal de Educação, Ciência e Tecnologia do Piauí. Campus Corrente. \\ Rua Projetada Trinta e Seis, 380. Nova Corrente, Corrente-PI, Brasil (CEP \\ 64980-000). \\ ${ }^{2}$ Instituto Federal de Educação, Ciência e Tecnologia do Piauí. Campus Teresina \\ Central. Rua Álvaro Mendes, 94. Centro. Teresina-PI, Brasil (CEP: 64000-040). \\ ${ }^{3}$ Universidade Federal Rural do Rio de Janeiro. Programa de Pós-Graduação em \\ Agronomia. BR-465, km 07. Zona Rural. Seropédica-RJ, Brasil (CEP 23890-000). \\ E mail: gutierailan@gmail.com. \\ ${ }^{4}$ Universidade Estadual de Goiás. Departamento de Agronomia. Campus Palmeira \\ de Goiás. Rua 7, S/№. Palmeira de Goiás-GO, Brasil (CEP 76190-000).
}

Resumo. O Cerrado Baiano ou Gerais Baiano, como é comumente citado, abrange importantes unidades territoriais do bioma, as bacias hidrográficas. Essas exercem grande papel ecológico, por serem as principais contribuintes dos afluentes da margem esquerda do Rio São Francisco, na qual garantem uma perene disponibilidade hídrica para múltiplos usos, com destaque para os projetos de irrigação, assim como algumas usinas hidroelétricas do nordeste brasileiro. Nesta perspectiva, a presente pesquisa objetivou especializar os níveis de vulnerabilidade ambiental decorrentes dos processos degradativos do solo na Bacia do Rio Preto, Estado da Bahia, Brasil. Para a determinação da vulnerabilidade dos solos, foram atribuídas notas de risco à degradação para cada um dos critérios (geomorfologia, pedologia, vegetação, geologia e clima, sendo então obtido o mapa de vulnerabilidade da bacia). Após o cálculo dos graus de vulnerabilidade, os dados foram especializados utilizando estatística multivariada pelo uso da Krigagem, por meio do software ArcGIS 10.3, permitindo a distribuição dos graus pelos solos da bacia. Este método permitiu agregar os valores dos componentes ambientais a área da bacia estudada, e desse modo, gerar uma espacialização dos níveis de vulnerabilidade pela área total. 0 estudo da espacialização dos graus de vulnerabilidade da Bacia do Rio Preto verificou um predomínio em $75 \%$ da área total da bacia, do grau de vulnerabilidade calculado de 2,0, decorrente dos elementos do ambiente, com influência predominante da tipologia fitofisionômica da bacia. A análise da vulnerabilidade ambiental
Recebido $07 / 07 / 2020$

Aceito

$30 / 05 / 2021$

Disponível on line $31 / 05 / 2021$

Publicado $31 / 08 / 2021$

Acesso aberto

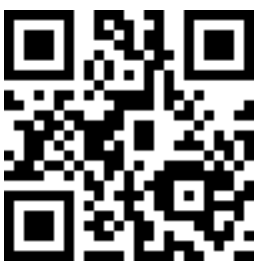


permitiu verificar que a Bacia do Rio Preto encontra-se, em sua maior parte, em situação medianamente/estável-vulnerável, o que se torna um fator preocupante, principalmente pelo desenvolvimento das áreas de pastagens e agricultura mecanizada por décadas inseridas nos locais de maior vulnerabilidade ambiental, principalmente nas zona ecotonal de maior susceptibilidade a erosão dos solos, que representam um predomínio em $75 \%$ da área total da bacia. 0 estudo da espacialização da vulnerabilidade da Bacia do Rio Preto utilizando o método de apresentou resultados que podem ser utilizados para estudos de planejamento relativos à questão espacial e ambiental da bacia.

Palavras-chave: Interpolação espacial; Uso e ocupação do solo; Solos vulneráveis.

\section{Abstract. Spatialization of the vulnerability level of the soils of the Rio Preto Watershead, Bahia, Brazil. The Cerrado Baiano or} Gerais Baiano, as it is commonly quoted, covers important territorial units of the biome, the basins. These have a great ecological role, as they are the main contributors of the tributaries of the left bank of the São Francisco River, in which they guarantee a perennial water availability for multiple uses, especially the irrigation projects, as well as some hydroelectric power plants in the Brazilian northeast. In this perspective, the present research aimed to specialize the levels of environmental vulnerability due to soil degradation processes in the Rio Preto Basin, State of Bahia, Brazil. For the determination of soil vulnerability, were assigned degradation risk notes for each of the following criteria (geomorphology, pedology, vegetation, geology and climate, and the vulnerability map of the basin) was obtained. After calculating the vulnerability levels, the data were specialized using multivariate statistics using Kriging, using the software ArcGIS 10.3, allowing the distribution of the degrees by the soils of the basin, the method allowed to aggregate the values of the environmental components to the area of the basin studied, thus generating spatialization of levels of vulnerability across the total area. The study of the spatialization of vulnerability degrees of the Rio Preto Basin found a predominance in $75 \%$ of the total area of the basin, the degree of vulnerability calculated of 2.0, due to the elements of the environment, with predominant influence of the phytophysiological typology of the basin. The analysis of the environmental vulnerability allowed to verify that the basin of the Rio Preto is, for the most part, in a moderately/ stable-vulnerable situation, which becomes a worrisome factor, mainly for the development of the pasture and mechanized agriculture for decades inserted in the places of greater environmental vulnerability, mainly in the ecotonal zone of greater susceptibility to the erosion of the soils, that represent a predominance in $75 \%$ of the total area of the basin. The study of

\footnotetext{
ORCID

(1) 0000-0002-0979-0657

Nayara Caroline

Moreira Leopoldo

(D) 0000-0002-6465-9731

Bruna de Freitas Iwata

(D) 0000-0002-0635-2651

Tancio Gutier Ailan

Costa

(D) 0000-0003-2029-9997

Juliana Vogado Coelho

(D) 0000-0001-5264-8499

Gleide Ellen dos

Santos Clementino

(D) 0000-0003-0099-2487

Cristian Epifânio de

Toledo
} 
the vulnerability spatialization of the Rio Preto Basin using the method presented results that can be used for planning studies related to the spatial and environmental issue of the basin.

Keywords: Spatial interpolation; Land use and occupation; Vulnerable soils.

\section{Introdução}

O Cerrado Baiano ou Gerais Baiano, como é comumente citado, abrange importantes unidades territoriais do bioma, corresponde as bacias hidrográficas. Estas, por sua vez, exercem grande papel ecológico, por serem as principais contribuintes dos afluentes da margem esquerda do Rio São Francisco, na qual garantem uma perene disponibilidade hídrica para múltiplos usos, com destaque para os projetos de irrigação e, algumas usinas hidroelétricas do nordeste brasileiro (Donagemma et al., 2008).

Com base em fundamentações de Donagemma et al. (2008), o conjunto de fatores edafoclimáticos disponíveis para região, associada aos baixos preços das áreas agricultáveis e disponibilidade hídrica o ano todo, em particular nas áreas de vegetação de Cerrado, promovem a rápida e grande expansão agrícola na região.

Em contraposição Freitas et al. (2004), mencionam que essas condicionantes, possuem como fatores limitantes para essa expansão, a grande diversidade pedoambiental (solos, vegetação e clima) que exerce influência direta de significativo risco para a agricultura de sequeiro, quando há usos sem estratégias de planejamento e/ou planejamento inadequado para o manuseio dessas terras. Como é o caso da crescente utilização de modernos sistemas de cultivo aliado ao aumento de ações de desmatamento desenfreado de áreas de recarga, remanescentes naturais e matas ciliares, causando danos ambientais adversos e tornando o solo lixiviado e menos permeável, e assim impedindo que o solo exerça o papel de filtro e de condutor.

Atrelado a isso, Goedert e Oliveira (2007), abordam que a utilização de manejo intensivo/inadequado do solo tende a resultar na diminuição de sua qualidade, com consequências ambientais, sociais e econômica. 0 que para Araújo e Monteiro (2007), a qualidade ambiental do solo, especialmente o solo do Cerrado Baiano, pode ser mensurada através da utilização de indicadores, que caracterizam o status ambiental ou a condição de conservação do ecossistema.

Neste sentido, diversos trabalhos têm se utilizado como indicadores da qualidade do solo para avaliar o cumprimento de suas funções ecológicas a partir de seus atributos químicos, físicos e biológicos. E com isso, considera-se uma seleção de indicadores da qualidade do solo e do ambiente, aplicados a modelos inseridos a softwares, e diagramas que integram os resultados quantitativamente e graficamente, capazes de abordar de forma integrada áreas possivelmente degradadas e/ou vulneráveis em unidades territoriais naturais, como as bacias hidrográficas. Nesse sentido, o trabalho teve como objetivo identificar os níveis de vulnerabilidade ambiental decorrentes aos processos de degradativos do solo na Bacia do rio Preto, Bahia.

\section{Metodologia}

\section{Caracterização da Bacia do Rio Preto}

A Bacia do Rio Preto ocupa toda a parte setentrional da Bacia do Rio Grande, sendo um dos principais contribuintes da margem esquerda da Bacia do Rio Grande, que por sua vez contribui diretamente com o Rio São Francisco. 0 Rio Preto atravessa os municípios 
baianos de Formosa do Rio Preto, Santa Rita de Cássia e Mansidão (Figura 1), desaguando no Rio Grande, próximo à Serra do Boqueirão, no Município de Mansidão.

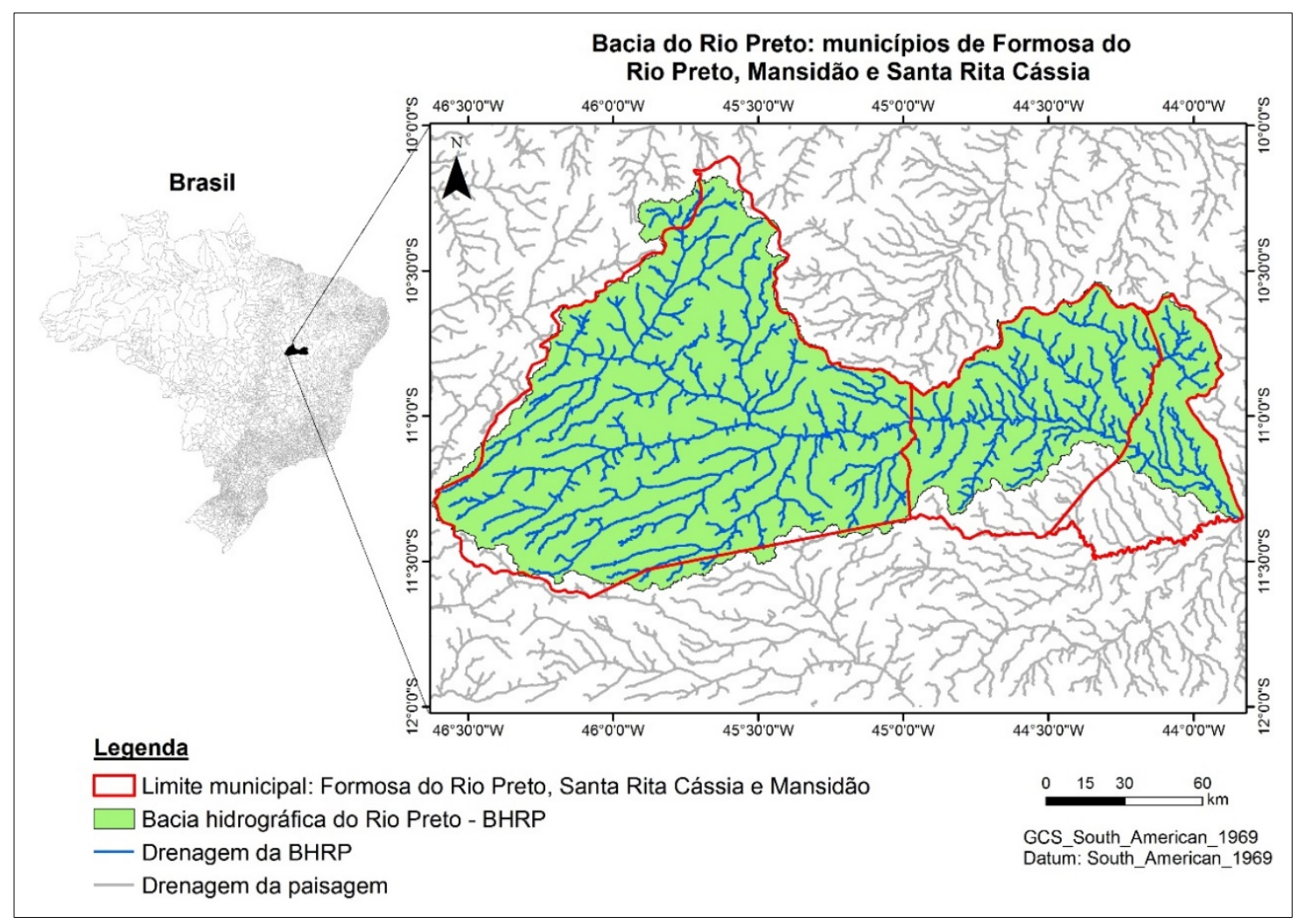

Figura 1. Localização da Bacia do Rio Preto, Bahia, Brasil.

A caracterização climática da região do Oeste Baiano propicia um importante papel na forma de uso e ocupação e manejo do solo, desde os primórdios até a sua situação atual. Tal característica climática, exerce uma influência direta nas atividades agrícolas da região, assim como no uso dos recursos hídricos. Segundo Gaspar (2006) o clima é classificado como um clima Tropical, com inverno seco - do tipo Aw, conforme classificação de Köppen. 0 clima semiúmido se apresenta no limite oeste do estado da Bahia, ao longo da cabeceira das sub bacias dos afluentes da margem esquerda do rio São Francisco, Corrente e Grande, abrangendo uma faixa vertical limitada ocidentalmente pelos Estados de Goiás e Tocantins (SRH, 2003).

\section{Vulnerabilidade ambiental}

0 estudo utilizou como método de determinação da vulnerabilidade ambiental a metodologia conforme Crepani et al. (2001), que consiste em apresentar um intervalo de valores de estabilidade/instabilidade (ou vulnerabilidade) distribuídos entre as situações de predomínio dos processos pedogenéticos (valores próximos de 1,0), passando por situações intermediárias (valores ao redor de 2,0) e situações de predomínio dos processos de morfogênese (valores próximos de 3,0). A partir disso, quantificou-se os graus de vulnerabilidade da bacia conforme tabela abaixo (Tabela 1). 
Tabela 1. Grau de vulnerabilidade calculado dos solos da bacia do Rio Preto, conforme parâmetro da cobertura vegetal

\begin{tabular}{lcc}
\hline Cobertura vegetal & Valor calculado & Grau de vulnerabilidade \\
\hline Cerrado & $\mathbf{V = 2 , 0}$ & Medianamente estável/vulnerável \\
Caatinga & $\mathbf{V = 2 , 0}$ & Medianamente estável/vulnerável \\
Remanescente de Floresta Mata Atlântica & $\mathbf{V = 1 , 4}$ & Medianamente estável \\
\hline
\end{tabular}

* $\mathrm{V}=(\mathrm{G}+\mathrm{R}+\mathrm{S}+\mathrm{Ve}+\mathrm{C}) / 5$ formulário para cálculo

Após cálculo dos graus de vulnerabilidade os dados foram especializados utilizando estatística multivariada pelo uso da Krigagem, por meio do software ArcGIS permitindo a distribuição dos graus pelos solos da bacia.

\section{Resultados e discussão}

O estudo da espacialização do grau de vulnerabilidade da Bacia do Rio Preto verificou um predomínio em $75 \%$ da área total da bacia, do grau de vulnerabilidade calculado de 2,0 (Crepani et al., 2001), decorrente dos elementos bióticos e abióticos que compõem a bacia, com influência predominante da tipologia fitofisionômica da região (Figura 2). Foi possível vincular o estudo ao comportamento mais sensível às alterações ambientais as áreas da bacia com cobertura vegetal tipicamente dos biomas Cerrado e Caatinga, no qual não foram feitas distinções no comportamento deles em relação ao grau de perda de qualidade ambiental.

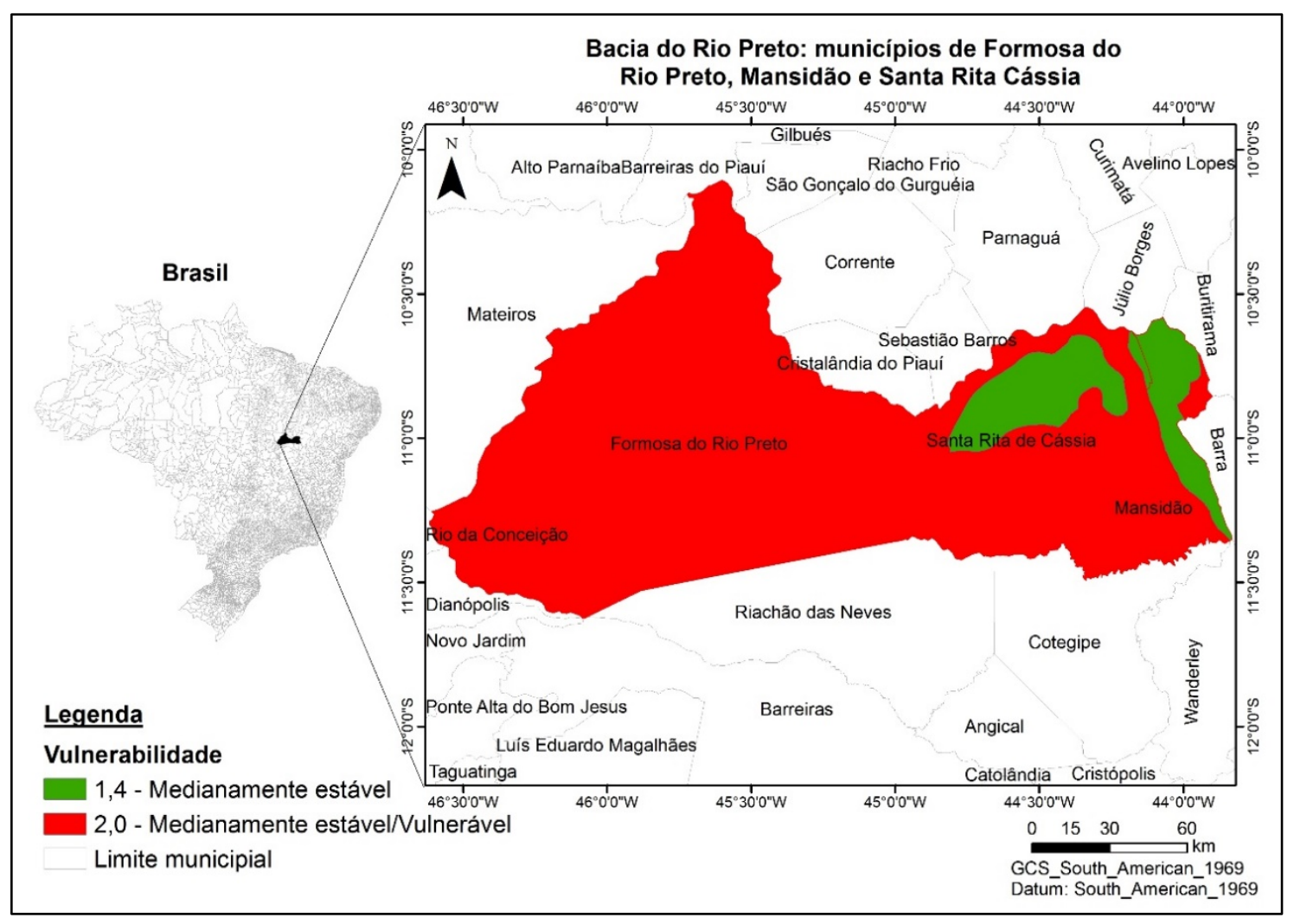

Figura 2. Mapa de vulnerabilidade ambiental da Bacia do Rio Preto, Bahia, Brasil. 
Em função das observações e interpretação dos dados obtidos, constatou-se uma correlação direta entre o uso e cobertura do solo de forma a minimizar impactos sobre a aceleração dos processos erosivos em bacias hidrográficas. Desse modo, fica evidente que enquanto houver existência de cobertura do solo por cobertura vegetal natural nas fitofisionomias da bacia, as demais variáveis determinantes da vulnerabilidade ambiental, (solo, relevo, geologia e clima) tendem a não se mostrar com tanta influência sobre os processos erosivos (Cunha et al., 2011).

A vulnerabilidade ambiental atrelada a indicadores socioambientais significativos, concomitantemente resulta em áreas suscetíveis à desertificação, o que caracteriza às regiões semiáridas e subúmidas seca, com a presença das vegetações típicas e predominantes nessas áreas que pertencem aos ecossistemas integrantes do bioma Caatinga e Cerrado correspondentes a grande parte do semiárido nordestino. Diante disso, é válido ressaltar que, o uso do solo desses biomas e suas respectivas fitofisionomias savânicas, são constituídas de pastagem plantada, e de áreas degradadas (erodidas, desertificadas e salinizadas) em extensão superior a 2,3 milhões de hectares, ou 3,5\% das terras degradadas do país (MCTI, 2016)

Nessas áreas, a cobertura vegetal natural de caráter lenhoso, conforme valoração econômica, é utilizada para produção de lenha e carvão vegetal, de modo que a extração da lenha se tornou a principal atividade econômica nessa região. Em contramão, o manejo inadequado da vegetação natural nessas áreas, implica em ameaça a continuidade da produção de bens e serviços ambientais dos remanescentes e ecossistemas da Caatinga, que já são prejudicados pelas adversidades climáticas naturais, como alta evapotranspiração, ocorrência de longos períodos de secas, solos rasos, alta salinidade, baixa fertilidade e reduzida capacidade de retenção de água, o que limita seu potencial produtivo, em função do uso inadequado dos recursos florestais do bioma (Paupitz, 2013).

Nesse contexto, a respeito a susceptibilidade a vulnerabilidade ambiental dos remanescentes de floresta de Marta Atlântica, Geneletti (2004) aborda que a fragmentação de ecossistemas, de maneira ampla, caracteriza-se pelos efeitos da diminuição em seus tamanhos, manejo inadequado, ausência de práticas conservacionistas, expansão de práticas agrícolas, e aumento da suscetibilidade a distúrbios externos, e/ou alterações em suas condições físicas, assim como intensa fragmentação que age tornando o ecossistema frágil. Segundo Viana (1990) o termo fragmento pode ser explicado como um processo dentro dos ecossistemas que interrompe as áreas por meio de barreiras antrópicas (estradas, pastagens) ou naturais (montanhas, lagos) com capacidade de reduzir o fluxo de animais, pólen ou sementes, de forma a exercer uma maior pressão aos fragmentos os tornando-os mais vulneráveis.

Quanto ao método para classificação da vulnerabilidade ambiental, o modelo aplicado enquadra duas fitofisionomias distintas em relação as principais condicionantes ambientais, como as espécies vegetais, considerando a densidade florestal do bioma Cerrado que tem em sua formação vegetacional determinada pela sazonalidade das chuvas, pelas diferenças na altitude, pela baixa fertilidade do solo, elevado potencial aquífero e elevada frequência de queimadas naturais (Barbosa e Schmiz 1998). Entretanto, embora o modelo tenha apontado o mesmo grau de vulnerabilidade para o bioma Cerado e Caatinga ressalta-se que os dois biomas possuem diferenças significativas na sua formação, que por sua vez diferem também quanto a adaptação climática, pois o Cerrado apresenta marcante sazonalidade climática, com precipitação média de $800 \mathrm{~mm}$ ou $\geq 800 \mathrm{~mm}$ anualmente, conforme a região.

0 efeito sazonal das chuvas é compensado pela extensa e irrigada malha hidrográfica do Cerrado (MCTI, 2016), que se diverge das condicionantes climáticas do bioma Caatinga pela ocorrência de secas estacionais estabelecendo regimes intermitentes aos cursos hídricos e contribuindo para que a vegetação perca as folhas como forma de subsistência, considerando relações ecológicas, entre as distintas fitofisionomia dos 
biomas Cerrado e Caatinga. No entanto, existe uma semelhança entre as duas fitofisionomias, por se encontrar em uma zona de transição, estas se presumem no comportamento da vegetação e condições climáticas aproximadas.

Sampaio (2010) descreve que a literatura se torna deficiente quando visa estabelecer critérios para as áreas de transição, onde não se distingue vegetação de caatinga das matas secas, no seu limite leste, e com as vegetações típicas de cerrado, principalmente no seu limite oeste. Embora como são misturas heterogêneas e descontínuas, prestam-se pouca atenção a determinação de limites, que tenham sido definidos por principalmente critérios climáticos. Nesse mesmo contexto, o autor explica que as médias de precipitação são maiores em zonas do topo das chapadas.

Farias e Castro (2004) conjugam os índices pluviométricos como abundantes e regulares contribuindo diretamente para tornar os solos menos férteis dando lugar a encraves de Cerrado e uma interpenetração complexa até o bioma Caatinga, em que os locais mais úmidos e férteis dessa zona ecotonal, transmuta-se em cerradões e matas de cocais.

0 médio e baixo curso da bacia tem como características ser considerada área de transição para o bioma Caatinga com 10,5\% de representatividade e Cerrado, em zonas físicas de relevo parcialmente ondulado (Silva, 2012). Assim quanto o uso e ocupação da bacia, tem-se a representatividade da atividade agropecuária como principal atividade econômica dos municípios de Santa Rita de Cássia e Mansidão, visto que as condições vigentes na área não favoreceram a implantação da agricultura mecanizada (Alves et al., 2011).

O estudo também verificou uma correlação entre as projeções de vulnerabilidade ambiental dos solos e os usos existentes nessa área (Figura 3), no qual apesar das intervenções antrópicas decorrentes do processo de exploração principalmente em consequência da exploração vinculadas as atividades do agronegócio, em produção de grãos em larga escala, a área da bacia ainda é predominantemente coberta por vegetação nativa remanescente. Identifica-se que nessas áreas que há correlação de relevo aplainado (Chapada) existe a presença de vegetação com aspectos que vai do Campo Sujo de Cerrado ao Chapadão, com vegetação densa de árvores e arbustos (Tschiedel, 2004).

Nota-se, no entanto, que a exploração agrícola da região tem favorecido significativamente o processo de depleção dos componentes naturais do ambiente. Esses usos intermitentes na bacia têm contribuído com a perda de qualidade ambiental dos solos da região, principalmente pelas etapas de desflorestamento, seguidas de uso exaustivo das áreas e adensamento pelo manejo de maquinário pesado. Mueller (1995), aponta que há ausência de sustentabilidade empregada nas tecnologias advindas da "Revolução Verde" e cita como tem provocado impactos por meio da mecanizada agricultura nos Cerrados. Alguns desses impactos são apontados por Cunha (1996) citar a compactação e impermeabilização dos solos pelo uso intensivo de máquinas agrícolas; processos erosivos; contaminação por agrotóxicos nos solos e cursos hídricos, perda de habitat natural pra fauna; impactos detrimentais da retirada da vegetação nativa de áreas contínuas extensas; assoreamento de reservatórios subterrâneos e susceptibilidade a pragas, como sendo um dos principais impactos nessas áreas. 


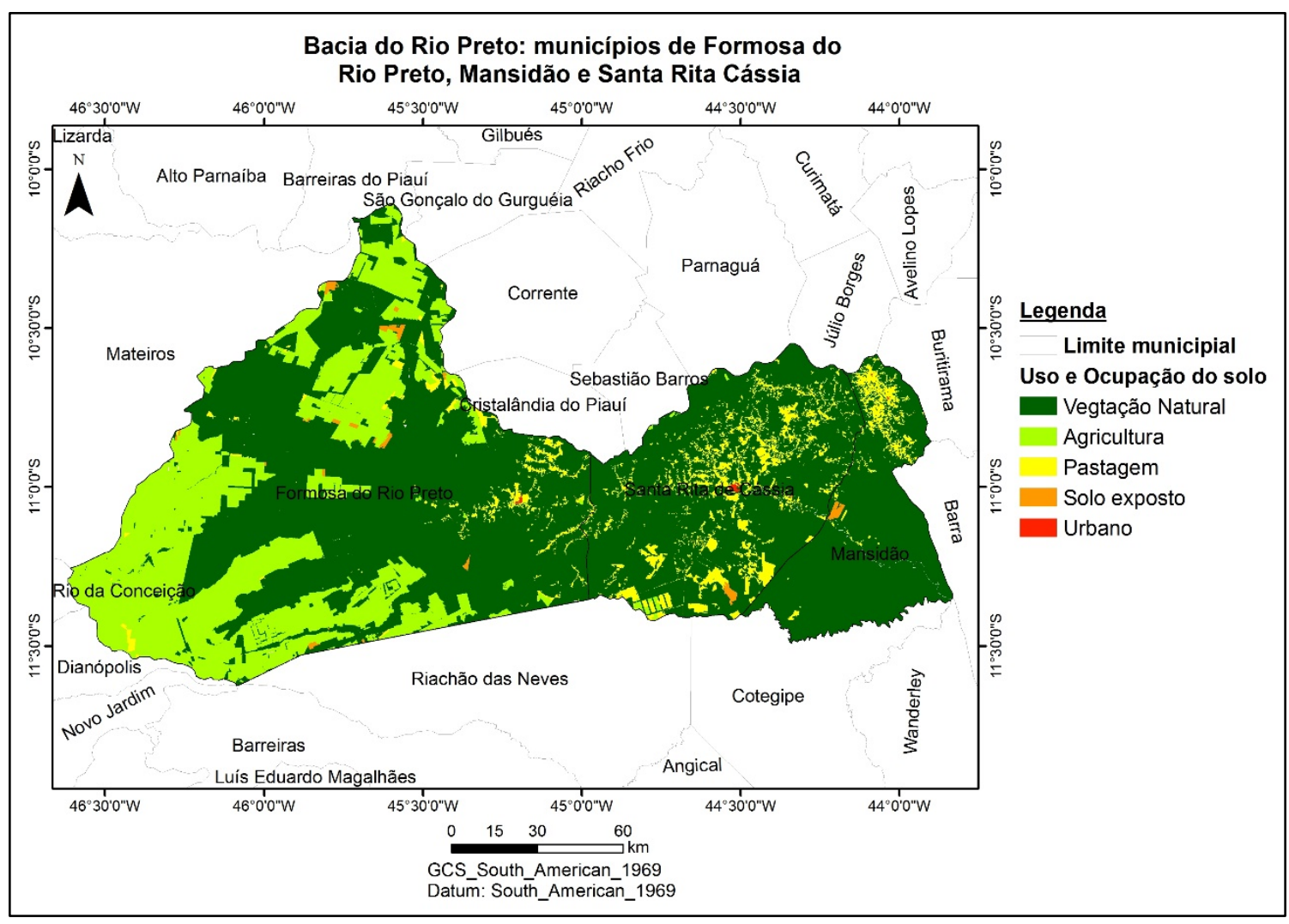

Figura 3. Espacialização do uso e ocupação dos solos da Bacia do Rio Preto, Bahia, Brasil.

Ademais, tem-se a exposição de áreas pelo uso e manejo de pastagens, principalmente de gado de corte nessas áreas (Figura 3). Essa exploração promove a perda de qualidade ambiental principalmente pelo manejo convencional adotado, visto que, as principais áreas de pastagens não possuem um manejo conservacionista no seu processo de exploração, ocorrendo de forma exaustiva, iniciada pelo desmate de vastas extensões de terra, sem reposição de biomassa orgânica, expondo assim os solos vulneráveis a perda de nutrientes, e ainda vulneráveis aos processos de compactação e alteração da estrutura física, pelo pisoteio animal.

Marchão et al. (2009) caracteriza as ações de pisoteio animal, como sendo atividade desencadeadora de compactação do solo, que se agrava pela remoção da vegetação pelo pastejo animal, que tende a diminuir as taxas de infiltração, e consequentemente desencadear e/ou aumentar os processos erosivos e reduzir o crescimento radicular das plantas nativas, deste modo fica notório os significativos impactos decorrentes do pisoteio e superpastoreio, exerce sobre as propriedades físicas, químicas e biológicas do solo.

Na espacialização dos usos e formas de ocupação dos solos da bacia também ficou evidente áreas de solo exposto (Figura 3). Em escala territorial esses espaços podem representar severas perdas de solo e acelerado processo de degradação, como voçorocamento e ravinas. A ocorrência de áreas com manifestação de alteração ambiental em elevado nível de perda de qualidade pode expressar o risco potencial que a região possui decorrente do grau de vulnerabilidade dos seus solos, enquadrado em $75 \%$ da área como grau 2,0 . 


\section{Conclusões}

A Bacia Hidrográfica do Rio Preto (Bahia) apresenta-se medianamente estável/vulnerável à perda de solos, estado condicionado por variáveis ambientais que integram a área, como a zona de transição dos biomas Cerrado e Caatinga, de fitofisionomias distintas, tendo o clima e o estágio de cobertura vegetal, importante atuação na perda da qualidade ambiental da área estudada.

0 estudo da espacialização da vulnerabilidade da Bacia do Rio Preto conforme o método utilizado apresentou resultados que podem ser utilizados para estudos de planejamento relativos à questão espacial e ambiental da bacia.

O mapa de vulnerabilidade ambiental funcionará para que o Comitê da Bacia Hidrográfica do rio Grande, ao qual a bacia hidrográfica do rio Preto faz parte, auxilie à administração, no planejamento do uso e ocupação do solo, visando à regularização do manejo agrícola nas áreas de maior vulnerabilidade.

\section{Conflito de interesses}

Os autores declaram não haver conflito de interesses.

\section{Referências}

Alves, R. R.; Brasil, J.; Meira, S. A.; Feitosa, G. D. Fragilidade ambiental na Bacia Hidrográfica do Rio Preto - Oeste da Bahia. In: Mondardo, M. L. (Org.). Espaços agrários e meio ambiente: Bahia, Bahias. Salvador: Ponto da Cultura, 2011. p. 204-222.

Araújo, A. S. F. Monteiro, R. T. R. Indicadores biológicos de qualidade do solo. Bioscience Journal, v. 23, n. 3, p. 66-75, 2007.

Barbosa, A. S.; Schmiz, P. I. Ocupação indígena do cerrado: esboço de uma história. In: Sano, S. M., Almeida, S. P. Cerrado: ambiente e flora. Planaltina: Embrapa, 1998. p. 3-42.

Crepani, E.; Medeiros, J. S.; Hernandez Filho, P.; Florenzano, T. G.; Duarte, V.; Barbosa, C. C. F. Sensoriamento remoto e geoprocessamento aplicados ao zoneamento ecológicoeconômico e ao ordenamento territorial. São José dos Campos: Instituto Nacional de Pesquisas Espaciais, 2001. (INPE-8454-RPQ/722).

Cunha, N. G.; Silveira, R. J. C.; Severo, C. R. S. Estudos dos solos do Município de Arroio Grande. Pelotas: Ed. UFPel, 1996.

Cunha, C. R. C.; Dupas, A. F.; Pons, D. A. N.; Tundisi, J. G. Análise da influência das variáveis ambientais utilizando inferência fuzzy e zoneamento das vulnerabilidades. Estudo do caso da Bacia Hidrográfica do Ribeirão do Feijão, São Carlos-SP. Geociências, v. 30, n. 3, p. 399414, 2011.

Donagemma, K. G.; Calderano, B.S.; Manzatto, V. C; Bernardi, A.; Gregoris, G.; Dowich, I. Comportamento físico-químico, aspectos mineralógicos e resposta ao manejo de Latossolos de textura média do Oeste da Bahia. Anais da XVIII Reunião Brasileira de Manejo e Conservação do Solo e da Água, Teresina, Embrapa Meio-Norte, Universidade Federal do Piauí, 2010.

Farias, R. R. S.; Castro, A. A. J. F. Fitossociologia de trechos da vegetação do Complexo de Campo Maior, Campo Maior, PI, Brasil. Acta Botanica Brasilica, v. 18, p. 949-963, 2004. https://doi.org/10.1590/S0102-33062004000400025 
Freitas, P. L.; Bernardi, A. C. C.; Manzatto, C. V.; Ramos, D. P.; Döwich, I.; Landers, J. N. Comportamento físico-químico dos solos de textura arenosa e média do oeste baiano. Rio de Janeiro: Embrapa Solos: 2004. (Comunicado técnico, 27).

Gaspar, M. T. P. Sistema Aquífero Urucuia: caracterização regional e proposta de gestão. Brasília: Universidade de Brasília, 2006. (Tese de doutorado).

Geneletti, D. Using spatial indicators and value functions to assess ecosystem fragmentation caused by linear infrastructures. International Journal of Applied Earth Observation and Geoinformation, v. 5, p. 1-15, 2004. https://doi.org/10.1016/ j.jag.2003.08.004

Goedert, W. J.; Oliveira, S. A. Fertilidade do solo e sustentabilidade da atividade agrícola. In: Novais, R. F.; Alvarez, V. V. H.; Barros, N. F.; Fontes, R. L.; Cantarutti, R. B.; Neves, J. C. L. (Eds.). Fertilidade do solo. Viçosa: SBCS, 2007. p. 991-1017.

Marchão, L. R.; Vilela, L.; Paludo, L. A.; Guimarães Júnior, R. Impacto do pisoteio animal na compactação do solo sob integração lavoura-pecuária no oeste baiano. Planaltina: EMBRAPA Cerrados, 2009.

MCTI - Ministério da Ciência, Tecnologia e Inovação. Secretaria de Políticas e Programas de Pesquisa e Desenvolvimento. Coordenação-Geral de Mudanças Globais de Clima. Brasília, 2016.

Mueller, C. C. Dinâmica, condicionantes e impactos sócio-ambientais da evolução da fronteira agrícola no Brasil. Rio de Janeiro: Instituto Sociedade, População e Natureza, 1995. (Documento de Trabalho, 7).

Paupitz, J. Versão Final do Relatório Nacional de Implementação da UNCCD. Brasília: Secretaria de Extrativismos e Desenvolvimento Rural Sustentável, 2013.

Sampaio, B.S. V.E. Uso e conservação dos recursos florestais da Caatinga: caracterização do Bioma Caatinga. Brasília: Ministério do Meio Ambiente, 2010.

SRH - Superintendência de Recursos Hídricos. Plano Estadual de Recursos Hídricos da Bahia (PERH-BA). Salvador: Magna/BRL Ingénierie, 2003.

Tschiedel, M. W. Aplicação de estudo geofísico como contribuição ao conhecimento da tectônica da Sub-Bacia Urucuia. Brasília: Universidade de Brasília, 2004. (Dissertação de mestrado).

Viana, V. M. Biologia e manejo de fragmentos florestais naturais. Anais do VI Congresso Florestal Brasileiro, Campos do Jordão, SBS, SBEF, p. 113-118, 1990.

Informação da Licença: Este é um artigo Open Access distribuído sob os termos da Licença Creative Commons Attribution, que permite uso irrestrito, distribuição e reprodução em qualquer meio, desde que a obra original seja devidamente citada. 